\title{
Mechanical power during extracorporeal membrane oxygenation and hospital mortality in patients with acute respiratory distress syndrome
}

\author{
Li-Chung Chiu' ${ }^{1,2,3}$, Shih-Wei Lin ${ }^{1}$, Li-Pang Chuang ${ }^{1}$, Hsin-Hsien Lii, ${ }^{4,5}$, Pi-Hua Liu ${ }^{6,7}$, Feng-Chun Tsai ${ }^{8}$, \\ Chih-Hao Chang ${ }^{1,3}$, Chen-Yiu Hung ${ }^{1,3}$, Chung-Shu Lee' ${ }^{1}$, Shaw-Woei Leu' ${ }^{1}$, Han-Chung Hu ${ }^{1,4,9}$, \\ Chung-Chi Huang ${ }^{1,4,9}$, Huang-Pin $\mathrm{Wu}^{10}$ and Kuo-Chin Kao ${ }^{1,4,9,11^{*}}$ (])
}

\begin{abstract}
Background: Mechanical power (MP) refers to the energy delivered by a ventilator to the respiratory system per unit of time. MP referenced to predicted body weight (PBW) or respiratory system compliance have better predictive value for mortality than MP alone in acute respiratory distress syndrome (ARDS). Our objective was to assess the potential impact of consecutive changes of MP on hospital mortality among ARDS patients receiving extracorporeal membrane oxygenation (ECMO).

Methods: We performed a retrospective analysis of patients with severe ARDS receiving ECMO in a tertiary care referral center in Taiwan between May 2006 and October 2015. Serial changes of MP during ECMO were recorded.

Results: A total of 152 patients with severe ARDS rescued with ECMO were analyzed. Overall hospital mortality was 53.3\%. There were no significant differences between survivors and nonsurvivors in terms of baseline values of MP or other ventilator settings. Cox regression models demonstrated that mean MP alone, MP referenced to PBW, and MP referenced to compliance during the first 3 days of ECMO were all independently associated with hospital mortality. Higher MP referenced to compliance (HR 2.289 [95\% Cl 1.214-4.314], $p=0.010$ ) was associated with a higher risk of death than MP itself (HR 1.060 [95\% Cl 1.018-1.104], $p=0.005$ ) or MP referenced to PBW (HR 1.004 [95\% Cl 1.0021.007], $p<0.001$ ). The 90-day hospital mortality of patients with high MP (>14.4 J/min) during the first 3 days of ECMO was significantly higher than that of patients with low MP ( $\leqq 14.4 \mathrm{~J} / \mathrm{min})(70.7 \% \mathrm{vs} .46 .8 \%, p=0.004)$, and the 90 -day hospital mortality of patients with high MP referenced to compliance $\left(>0.53 \mathrm{~J} / \mathrm{min} / \mathrm{ml} / \mathrm{cm} \mathrm{H}_{2} \mathrm{O}\right)$ during the first 3 days of ECMO was significantly higher than that of patients with low MP referenced to compliance $(\leqq 0.53 \mathrm{~J} / \mathrm{min} / \mathrm{ml} /$ $\left.\mathrm{cm} \mathrm{H}_{2} \mathrm{O}\right)(63.6 \%$ vs. $29.7 \%, p<0.001)$.
\end{abstract}

Conclusions: MP during the first 3 days of ECMO was the only ventilatory variable independently associated with 90-day hospital mortality, and MP referenced to compliance during ECMO was more predictive for mortality than was MP alone.

\footnotetext{
*Correspondence: kck0502@cgmh.org.tw

1 Department of Thoracic Medicine, Chang Gung Memorial Hospital,

Chang Gung University College of Medicine, Linkou, No. 5, Fu-Shing St.,

GuiShan, Taoyuan, Taiwan

Full list of author information is available at the end of the article
} permits use, sharing, adaptation, distribution and reproduction in any medium or format, as long as you give appropriate credit to the original author(s) and the source, provide a link to the Creative Commons licence, and indicate if changes were made. The images or other third party material in this article are included in the article's Creative Commons licence, unless indicated otherwise in a credit line to the material. If material is not included in the article's Creative Commons licence and your intended use is not permitted by statutory regulation or exceeds the permitted use, you will need to obtain permission directly from the copyright holder. To view a copy of this licence, visit http://creativecommons.org/licenses/by/4.0/. The Creative Commons Public Domain Dedication waiver (http://creativeco mmons.org/publicdomain/zero/1.0/) applies to the data made available in this article, unless otherwise stated in a credit line to the data. 
Keywords: Mechanical power, Acute respiratory distress syndrome, Extracorporeal membrane oxygenation, Ventilator-induced lung injury, Functional lung size, Compliance, Mortality

\section{Background}

Mechanical ventilation remains the cornerstone of management strategies for acute respiratory distress syndrome (ARDS), and extracorporeal membrane oxygenation (ECMO) is widely used as a salvage therapy for refractory hypoxemia in patients with severe ARDS. ECMO allows the lungs to rest and prevents the risk of ventilator-induced lung injury (VILI) by lowering airway pressure, tidal volume $\left(V_{\mathrm{T}}\right)$, and $\mathrm{FiO}_{2}$. However, the optimal ventilation strategies for patients with severe ARDS receiving ECMO have yet to be defined $[1,2]$.

Mechanical power (MP) refers to the amount of energy per unit of time transmitted to the respiratory system by a mechanical ventilator, as determined by volume, pressure, flow, and respiratory rate (RR). It is therefore reasonable to assume that MP is superior to single ventilator parameter in predicting the risk of VILI [3, 4]. VILI originates from the interaction between the energy load (i.e., MP) and the pathophysiological characteristics of the lungs (size, homogeneity and recruitability) [4-6]. Therefore, the same MP may have different impact on respiratory system depending on the applied conditions of lungs, and MP should be referenced at least to the functional lung size in order to accurately reflect the actual amount of energy applied to the lungs, i.e., specific power [7-11].

Recent studies have shown that MP is independently associated with in-hospital mortality among critically ill patients [12], and high MP levels have been linked to increased mortality in ARDS patients [13]. However, MP alone does not have better predictive value for patients with ARDS, and it is preferable to adjust MP to predicted body weight (PBW) [7] or respiratory system compliance in terms of well-aerated tissue [8].

ECMO enhanced lung-protective ventilation to mitigate the energy load (i.e., MP) delivered to the respiratory system; however, researchers have yet to contrast the influence of MP alone and MP referenced to functional lung size on the mortality in ARDS patients undergoing ECMO. Our objective in this study was to assess the role of serial changes in MP (adjusted for PBW or compliance) on hospital mortality in patients with severe ARDS undergoing ECMO.

\section{Methods \\ Study design and patients}

This study was based on retrospective analysis of patients with severe ARDS who had been treated using ECMO between May 2006 and October 2015 at Chang Gung Memorial Hospital (CGMH) in Taiwan. CGMH is a tertiary care referral center with a 3700 -bed general ward and 278-bed adult intensive care unit (ICU) with a high volume of venoarterial and venovenous mode ECMO exceeding 100 cases annually, and only $20 \%$ of the indications for ECMO was patients with severe ARDS. Exclusion criteria were as follows: (1) age $<20$ years, (2) malignancies with poor prognosis within 5 years, (3) significant underlying comorbidities or severe multiple organ failure refractory to treatment, and (4) mortality within 3 days after ECMO initiation. The local Institutional Review Board for Human Research approved this study (CGMH IRB No. 201600632B0) and waived the need for informed consent.

\section{Definitions}

ARDS was defined in accordance with the Berlin criteria [14]. MP was calculated in accordance with the methods [4] based on $V_{\mathrm{T}}$, RR, peak inspiratory pressure (Ppeak), and driving pressure $(\Delta P)$ using the following equation:

$$
\begin{aligned}
\mathrm{MP}(\text { Joules } / \text { minutes })(\mathrm{J} / \mathrm{min})= & 0.098 \times V_{\mathrm{T}} \times \mathrm{RR} \\
& \times(\text { Ppeak }-1 / 2 \times \Delta P) . \\
\text { MP referenced to } \mathrm{PBW}= & \mathrm{MP} / \mathrm{PBW} . \\
\text { MP referenced to compliance }= & \mathrm{MP} / \text { Compliance. }
\end{aligned}
$$

Ppeak is equivalent to plateau pressure in pressurecontrolled ventilation [15-18]. Ppeak has been used as a surrogate for plateau pressure to calculate MP if not specified [19], and similar effect of MP on mortality was demonstrated when considering Ppeak instead of plateau pressure for calculating MP [12]. One recent prospective study used dynamic driving pressure (Ppeak minus PEEP) to calculate MP, referring to the measure as dynamic MP [20]. Hospital mortality refers to all-cause death during the hospital stay. Patients who remained alive for 90 days after discharge from the hospital were regarded as survivors.

\section{Data collection}

Demographic data, risk factors for ARDS, underlying comorbidities, Sequential Organ Failure Assessment 
(SOFA) score, and lung injury score were collected prior to ECMO initiation. The dates of hospital and ICU admission, ARDS onset, mechanical ventilator initiation and liberation, ECMO cannulation and decannulation, ICU and hospital discharge, and time of death were recorded. Arterial blood gas parameters and mechanical ventilator settings were recorded at the time of ECMO initiation and at approximately 10 a.m. on days 1,2 , and 3 after ECMO initiation.

\section{Statistical analysis}

Continuous variables were presented as mean \pm standard deviation or median (interquartile range), and categorical variables were reported as numbers (percentages). A student's $t$ test or the Mann-Whitney $U$ test was used to compare continuous variables between groups. Categorical variables were tested using the chi-square test for equal proportions or Fisher's exact test. Paired Student's $t$ tests were used to compare continuous variables before and after ECMO. Receiver operating characteristic curve and Youden index were used to determine the cutoff to dichotomize continuous variables. Risk factors associated with hospital mortality were analyzed using univariate analysis in the first step, followed by Cox proportional hazard regression model with stepwise selection. The results were presented using the hazard ratio (HR) and 95\% confidence interval (CI). Cumulative mortality curves were generated as a function of time using the Kaplan-Meier approach and compared using the log-rank test. All statistical analysis was performed using SPSS 22.0 statistical software, and a two-sided $p$ value $<0.05$ was considered statistically significant.

\section{Results}

A total of 152 patients with severe ARDS rescued by ECMO were included in the analysis, which examined the impact of MP on hospital mortality. Overall all-cause in-hospital mortality was $53.3 \%$. All patients were deeply sedated and paralyzed, and most cases received pressurecontrolled ventilation until attempts at weaning from ECMO. The ECMO techniques didn't show significant difference during the study period. Hospital mortality was not significantly different between patients in the earlier years and later years of the study period (20062011: 77 patients, mortality rate $54.5 \%$; 2012-2015: 75 patients, mortality rate $52 \%, p=0.753)$. Patients in the later years received significantly lower $V_{\mathrm{T}}$, higher PEEP, lower Ppeak, and lower MP during the first 3 days of ECMO than did patients in the earlier years (Additional file 1: Table S1). The mean value of MP from day 1 to day 3 on ECMO didn't show significant difference $(p=0.150)$, and mean MP during the first 3 days of ECMO was used to evaluate the impact on hospital mortality (Additional file 2: Table S2).

\section{Comparisons of survivors and nonsurvivors}

As shown in Table 1, the mean age of nonsurvivors was higher than that of survivors. Nonsurvivors suffered from ARDS for a longer duration before ECMO initiation, and a higher percentage were immunocompromised. There were no significant differences between the two groups in terms of baseline ventilator settings. After receiving ECMO support, nonsurvivors received significantly higher MP than did survivors, with higher MP referenced to $\mathrm{PBW}$, higher MP referenced to compliance, higher Ppeak, lower dynamic compliance, and higher total RR (all $p<0.05$ ). The SOFA scores of nonsurvivors were also significantly higher during the first 3 days of ECMO support.

\section{Comparing patients receiving high and low mechanical power}

As shown in Table 2, the maximum Youden index value was used to categorize patients according to MP, using a cutoff point of $14.4 \mathrm{~J} / \mathrm{min}$ during the first 3 days of ECMO: high MP group (41 patients; 27\%) and low MP group (111 patients; 73\%). No significant differences were observed between the two groups in terms of MP or other ventilator settings variables prior to ECMO initiation. After ECMO support, the high MP and low MP groups differed significantly in all ventilator settings variables except for PEEP and dynamic compliance (all $p<0.001$ ). Patients in the high MP group had significantly higher mortality than did patients in the low MP group. As shown in Table 3, the maximum Youden index value was used to categorize patients according to MP referenced to compliance, using a cutoff point of $0.53 \mathrm{~J} /$ $\mathrm{min} / \mathrm{ml} / \mathrm{cm} \mathrm{H}_{2} \mathrm{O}$ during the first 3 days of ECMO: high $\mathrm{MP} /$ Compliance group (88 patients; $58 \%$ ) and low MP/ Compliance group (64 patients; 42\%). Before ECMO initiation, MP/Compliance, not MP alone, was significantly different between the two groups. After ECMO support, the high MP/Compliance and low MP/Compliance groups differed significantly in all ventilator settings variables except for tidal volume. Patients in the high $\mathrm{MP} /$ Compliance group had significantly higher mortality than did patients in the low MP/Compliance group.

\section{Percentage changes in MP and its components after ECMO and correlation between MP and mortality}

Following ECMO initiation, there was a significant reduction in MP among the overall population (49\%, from 23.8 to $12.1 \mathrm{~J} / \mathrm{min}, p<0.001$ ), survivors $(55 \%$, from 24.1 to $10.9 \mathrm{~J} / \mathrm{min}, p<0.001)$, and nonsurvivors $(44 \%$, from 23.5 to $13.1 \mathrm{~J} / \mathrm{min}, p<0.001)$. Following ECMO 
Table 1 Background characteristics and clinical variables: survivors and nonsurvivors

\begin{tabular}{|c|c|c|c|c|}
\hline Variables & $\begin{array}{l}\text { All } \\
(n=152)\end{array}$ & $\begin{array}{l}\text { Survivors } \\
(n=71)\end{array}$ & $\begin{array}{l}\text { Nonsurvivors } \\
(n=81)\end{array}$ & $p$ \\
\hline Age (years) & $50.3 \pm 16.4$ & $46.0 \pm 16.5$ & $54.1 \pm 15.4$ & 0.002 \\
\hline Male (gender) & $103(67.8 \%)$ & $48(67.6 \%)$ & $55(67.9 \%)$ & 0.969 \\
\hline Body mass index $\left(\mathrm{kg} / \mathrm{m}^{2}\right)$ & $25.8 \pm 5.3$ & $26.0 \pm 5.8$ & $25.6 \pm 4.7$ & 0.631 \\
\hline \multicolumn{5}{|l|}{ ARDS etiologies } \\
\hline Pulmonary cause & $118(78 \%)$ & $59(83 \%)$ & $59(73 \%)$ & 0.130 \\
\hline Extrapulmonary cause & $34(22 \%)$ & $12(17 \%)$ & $22(27 \%)$ & 0.130 \\
\hline Diabetes mellitus & $40(26 \%)$ & $23(32 \%)$ & $17(21 \%)$ & 0.111 \\
\hline Chronic liver disease & $21(14 \%)$ & $6(9 \%)$ & $15(19 \%)$ & 0.073 \\
\hline Immunocompromised status & $40(26 \%)$ & $11(16 \%)$ & $29(36 \%)$ & 0.005 \\
\hline SOFA score before ECMO & $10.8 \pm 3.2$ & $10.3 \pm 3.1$ & $11.3 \pm 3.2$ & 0.067 \\
\hline Lung injury score before ECMO & $3.4 \pm 0.4$ & $3.4 \pm 0.4$ & $3.3 \pm 0.4$ & 0.106 \\
\hline ARDS duration before ECMO (h) & $28(7-122)$ & $10(4-64)$ & $54(17-195)$ & $<0.001$ \\
\hline $\mathrm{PaO}_{2} / \mathrm{FiO}_{2}(\mathrm{~mm} \mathrm{Hg})$ before ECMO & $63(52-88)$ & $64(53-80)$ & $63(52-107)$ & 0.168 \\
\hline \multicolumn{5}{|l|}{ Ventilator settings before ECMO } \\
\hline $\mathrm{MP}(\mathrm{J} / \mathrm{min})$ & $23.8 \pm 9.6$ & $24.1 \pm 10.3$ & $23.5 \pm 9.0$ & 0.668 \\
\hline $\mathrm{MP} / \mathrm{PBW}\left(\times 10^{-3} \mathrm{~J} / \mathrm{min} / \mathrm{kg}\right)$ & $416 \pm 172$ & $410 \pm 174$ & $423 \pm 171$ & 0.645 \\
\hline MP/Compliance $\left(\mathrm{J} / \mathrm{min} / \mathrm{ml} / \mathrm{cm} \mathrm{H}_{2} \mathrm{O}\right)$ & $1.27 \pm 0.76$ & $1.21 \pm 0.75$ & $1.33 \pm 0.78$ & 0.380 \\
\hline Tidal volume (ml/kg PBW) & $7.7 \pm 2.4$ & $7.7 \pm 2.3$ & $7.8 \pm 2.5$ & 0.658 \\
\hline $\operatorname{PEEP}\left(\mathrm{cm} \mathrm{H}_{2} \mathrm{O}\right)$ & $12.0 \pm 2.8$ & $12.2 \pm 2.5$ & $11.8 \pm 3.0$ & 0.288 \\
\hline Peak inspiratory pressure $\left(\mathrm{cm} \mathrm{H}_{2} \mathrm{O}\right)$ & $33.9 \pm 6.5$ & $33.6 \pm 6.0$ & $34.2 \pm 6.9$ & 0.605 \\
\hline Mean airway pressure $\left(\mathrm{cm} \mathrm{H}_{2} \mathrm{O}\right)$ & $18.6 \pm 4.4$ & $18.4 \pm 4.2$ & $18.8 \pm 4.6$ & 0.588 \\
\hline Dynamic compliance $\left(\mathrm{ml} / \mathrm{cm} \mathrm{H}_{2} \mathrm{O}\right)$ & $22.6 \pm 11.3$ & $23.7 \pm 11.6$ & $21.8 \pm 11.1$ & 0.420 \\
\hline Total respiratory rate (breaths/min) & $24.0 \pm 6.9$ & $23.7 \pm 7.4$ & $24.3 \pm 6.6$ & 0.596 \\
\hline Spontaneous respiratory rate (breaths/min) & $0(0-7)$ & $1(0-6)$ & $0(0-7)$ & 0.982 \\
\hline Minute ventilation (L/min) & $10.6 \pm 3.8$ & $10.7 \pm 4.1$ & $10.5 \pm 3.6$ & 0.816 \\
\hline SOFA score from day 1 to day 3 on ECMO & $9.6 \pm 2.3$ & $8.8 \pm 1.9$ & $10.4 \pm 2.4$ & $<0.001$ \\
\hline $\mathrm{PaO}_{2} / \mathrm{FiO}_{2}(\mathrm{~mm} \mathrm{Hg})$ from day 1 to day 3 on ECMO & $178(131-240)$ & $200(146-247)$ & $165(124-211)$ & 0.588 \\
\hline \multicolumn{5}{|l|}{ Ventilator settings from day 1 to day 3 on ECMO } \\
\hline $\mathrm{MP}(\mathrm{J} / \mathrm{min})$ & $12.1 \pm 6.2$ & $10.9 \pm 4.3$ & $13.1 \pm 7.4$ & 0.022 \\
\hline $\mathrm{MP} / \mathrm{PBW}\left(\times 10^{-3} \mathrm{~J} / \mathrm{min} / \mathrm{kg}\right)$ & $206 \pm 111$ & $185 \pm 67$ & $226 \pm 137$ & 0.022 \\
\hline MP/Compliance $\left(\mathrm{J} / \mathrm{min} / \mathrm{ml} / \mathrm{cm} \mathrm{H}_{2} \mathrm{O}\right)$ & $0.73 \pm 0.46$ & $0.60 \pm 0.32$ & $0.86 \pm 0.53$ & $<0.001$ \\
\hline Tidal volume (ml/kg PBW) & $6.0 \pm 2.2$ & $6.1 \pm 2.0$ & $6.0 \pm 2.4$ & 0.914 \\
\hline $\operatorname{PEEP}\left(\mathrm{cm} \mathrm{H}_{2} \mathrm{O}\right)$ & $12.0 \pm 3.3$ & $12.3 \pm 3.2$ & $11.7 \pm 3.3$ & 0.202 \\
\hline Peak inspiratory pressure $\left(\mathrm{cm} \mathrm{H}_{2} \mathrm{O}\right)$ & $31.7 \pm 5.6$ & $30.6 \pm 5.1$ & $32.8 \pm 5.9$ & 0.018 \\
\hline Mean airway pressure $\left(\mathrm{cm} \mathrm{H}_{2} \mathrm{O}\right)$ & $17.7 \pm 4.0$ & $17.4 \pm 3.6$ & $17.9 \pm 4.3$ & 0.406 \\
\hline Dynamic compliance $\left(\mathrm{ml} / \mathrm{cm} \mathrm{H}_{2} \mathrm{O}\right)$ & $19.2 \pm 8.1$ & $21.1 \pm 7.7$ & $17.4 \pm 8.1$ & 0.006 \\
\hline Total respiratory rate (breaths/min) & $16.0 \pm 4.4$ & $15.2 \pm 4.1$ & $16.7 \pm 4.6$ & 0.035 \\
\hline Spontaneous respiratory rate (breaths/min) & $1(0-4)$ & $0(0-4)$ & $2(0-5)$ & 0.114 \\
\hline Minute ventilation (L/min) & $5.7 \pm 2.8$ & $5.2 \pm 2.0$ & $6.0 \pm 3.2$ & 0.068 \\
\hline
\end{tabular}

Data are presented as mean \pm standard deviation, count or median (interquartile range)

$A R D S$ acute respiratory distress syndrome, $E C M O$ extracorporeal membrane oxygenation, $\mathrm{FiO}_{2}$ fraction of inspired oxygen, $\mathrm{MP}$ mechanical power, $\mathrm{PaO} \mathrm{O}_{2}$ partial pressure of oxygen in arterial blood, PBW predicted body weight, PEEP positive end-expiratory pressure, SOFA Sequential Organ Failure Assessment

initiation, there was a pronounced decrease in total $\mathrm{RR}$ and $V_{\mathrm{T}}(33 \%$ and $22 \%$, respectively, $p<0.001)$ with a less pronounced decrease in Ppeak (6\%) and no change in PEEP in the overall population (Fig. 1). Hospital mortality was correlated with MP during the first 3 days of
ECMO but not with the initial MP value before ECMO, and MP higher than $15.0 \mathrm{~J} / \mathrm{min}$ during the first 3 days of ECMO showed consistently increasing trends in mortality. The hospital mortality was $89 \%$ among patients with MP exceeding $20 \mathrm{~J} / \mathrm{min}$ during the first 3 days of ECMO 
Table 2 Ventilator settings, clinical variables, and outcomes as a function of mechanical power during ECMO

\begin{tabular}{|c|c|c|c|}
\hline \multirow[t]{2}{*}{ Variables } & \multicolumn{2}{|c|}{ MP during the first 3 days of ECMO } & \multirow[t]{2}{*}{$p$} \\
\hline & $\begin{array}{l}\text { High }(n=41) \\
(>14.4 \mathrm{~J} / \mathrm{min})\end{array}$ & $\begin{array}{l}\text { Low }(n=111) \\
(\leq 14.4 \mathrm{~J} / \mathrm{min})\end{array}$ & \\
\hline \multicolumn{4}{|l|}{ Ventilator settings before ECMO } \\
\hline $\mathrm{MP}(\mathrm{J} / \mathrm{min})$ & $25.0 \pm 9.5$ & $23.3 \pm 9.5$ & 0.339 \\
\hline $\mathrm{MP} / \mathrm{PBW}\left(\times 10^{-3} \mathrm{~J} / \mathrm{min} / \mathrm{kg}\right)$ & $441 \pm 166$ & $408 \pm 172$ & 0.316 \\
\hline MP/Compliance $\left(\mathrm{J} / \mathrm{min} / \mathrm{ml} / \mathrm{cm} \mathrm{H}_{2} \mathrm{O}\right)$ & $1.32 \pm 0.71$ & $1.26 \pm 0.78$ & 0.672 \\
\hline Tidal volume (ml/kg PBW) & $8.3 \pm 2.3$ & $7.5 \pm 2.4$ & 0.062 \\
\hline PEEP $\left(\mathrm{cm} \mathrm{H}_{2} \mathrm{O}\right)$ & $11.9 \pm 2.7$ & $12.0 \pm 2.8$ & 0.786 \\
\hline Peak inspiratory pressure $\left(\mathrm{cm} \mathrm{H}_{2} \mathrm{O}\right)$ & $34.4 \pm 6.5$ & $33.8 \pm 6.5$ & 0.568 \\
\hline Mean airway pressure $\left(\mathrm{cm} \mathrm{H}_{2} \mathrm{O}\right)$ & $19.2 \pm 3.9$ & $18.4 \pm 4.5$ & 0.310 \\
\hline Dynamic compliance $\left(\mathrm{ml} / \mathrm{cm} \mathrm{H}_{2} \mathrm{O}\right)$ & $22.3 \pm 8.4$ & $22.7 \pm 12.1$ & 0.869 \\
\hline Total respiratory rate (breaths/min) & $23.9 \pm 6.7$ & $24.0 \pm 7.1$ & 0.891 \\
\hline Spontaneous respiratory rate (breaths/min) & $1(0-6)$ & $0(0-7)$ & 0.956 \\
\hline Minute ventilation (L/min) & $11.2 \pm 3.5$ & $10.3 \pm 3.9$ & 0.205 \\
\hline \multicolumn{4}{|l|}{ Arterial blood gas before ECMO } \\
\hline $\mathrm{pH}$ & $7.24 \pm 0.16$ & $7.29 \pm 0.13$ & 0.056 \\
\hline $\mathrm{PaCO}_{2}(\mathrm{~mm} \mathrm{Hg})$ & $56.1 \pm 20.0$ & $51.1 \pm 18.4$ & 0.150 \\
\hline $\mathrm{PaO}_{2}(\mathrm{~mm} \mathrm{Hg})$ & $72.4 \pm 33.4$ & $74.5 \pm 41.7$ & 0.776 \\
\hline Saturation (\%) & $83.2 \pm 17.4$ & $85.1 \pm 14.4$ & 0.508 \\
\hline $\mathrm{PaO}_{2} / \mathrm{FiO}_{2}(\mathrm{~mm} \mathrm{Hg})$ & $66.5(49.7-85.7)$ & $63(53-90.7)$ & 0.882 \\
\hline SOFA score before ECMO & $11.9 \pm 3.1$ & $10.4 \pm 3.1$ & 0.013 \\
\hline \multicolumn{4}{|l|}{ Ventilator settings from day 1 to day 3 on ECMO } \\
\hline $\mathrm{MP}(\mathrm{J} / \mathrm{min})$ & $20.3 \pm 5.3$ & $9.1 \pm 3.0$ & $<0.001$ \\
\hline $\mathrm{MP} / \mathrm{PBW}\left(\times 10^{-3} \mathrm{~J} / \mathrm{min} / \mathrm{kg}\right)$ & $343 \pm 117$ & $159 \pm 55$ & $<0.001$ \\
\hline MP/Compliance $\left(\mathrm{J} / \mathrm{min} / \mathrm{ml} / \mathrm{cm} \mathrm{H}_{2} \mathrm{O}\right)$ & $1.14 \pm 0.48$ & $0.59 \pm 0.35$ & $<0.001$ \\
\hline Tidal volume (ml/kg PBW) & $7.4 \pm 2.2$ & $5.6 \pm 2.0$ & $<0.001$ \\
\hline PEEP $\left(\mathrm{cm} \mathrm{H}_{2} \mathrm{O}\right)$ & $11.8 \pm 2.5$ & $12.0 \pm 3.5$ & 0.653 \\
\hline Peak inspiratory pressure $\left(\mathrm{cm} \mathrm{H}_{2} \mathrm{O}\right)$ & $35.2 \pm 5.4$ & $30.5 \pm 5.1$ & $<0.001$ \\
\hline Mean airway pressure $\left(\mathrm{cm} \mathrm{H}_{2} \mathrm{O}\right)$ & $19.6 \pm 3.8$ & $17.0 \pm 3.8$ & $<0.001$ \\
\hline Dynamic compliance $\left(\mathrm{ml} / \mathrm{cm} \mathrm{H}_{2} \mathrm{O}\right)$ & $19.9 \pm 6.5$ & $18.9 \pm 8.5$ & 0.520 \\
\hline Total respiratory rate (breaths/min) & $20.3 \pm 5.4$ & $14.4 \pm 3.5$ & $<0.001$ \\
\hline Spontaneous respiratory rate (breaths/min) & $4(1-9)$ & $0(0-3)$ & $<0.001$ \\
\hline Minute ventilation (L/min) & $8.9 \pm 2.5$ & $4.5 \pm 1.6$ & $<0.001$ \\
\hline \multicolumn{4}{|l|}{ Arterial blood gas from day 1 to day 3 on ECMO } \\
\hline $\mathrm{pH}$ & $7.42 \pm 0.08$ & $7.44 \pm 0.08$ & 0.286 \\
\hline $\mathrm{PaCO}_{2}(\mathrm{~mm} \mathrm{Hg})$ & $38.6 \pm 6.5$ & $38.1 \pm 4.7$ & 0.639 \\
\hline $\mathrm{PaO}_{2}(\mathrm{~mm} \mathrm{Hg})$ & $102.2 \pm 65.9$ & $96.1 \pm 39.5$ & 0.489 \\
\hline Saturation (\%) & $94.8 \pm 3.3$ & $95.5 \pm 2.9$ & 0.240 \\
\hline $\mathrm{PaO}_{2} / \mathrm{FiO}_{2}(\mathrm{~mm} \mathrm{Hg})$ & $151(123-212)$ & $189(140-242)$ & 0.921 \\
\hline SOFA score from day 1 to day 3 on ECMO & $10.7 \pm 2.2$ & $9.2 \pm 2.2$ & 0.001 \\
\hline ECMO complications, $n$ (\%) & $9(22 \%)$ & $34(30.6 \%)$ & 0.292 \\
\hline Duration of ECMO (days) & $7.7(4.7-11.5)$ & $9.9(5.9-15.8)$ & 0.287 \\
\hline Duration of mechanical ventilator (days) & $15.4(11.8-34)$ & $22.9(12.4-39.8)$ & 0.291 \\
\hline Length of ICU stay (days) & $19(10-43)$ & $27(16-43)$ & 0.182 \\
\hline Length of hospital stay (days) & $29(13-63)$ & $41(24-65.5)$ & 0.130 \\
\hline ECMO-free days on day 28 & $0(0-18.2)$ & $10.1(0-19.3)$ & 0.075 \\
\hline Ventilator-free days on day 28 & $0(0-0)$ & $0(0-8.5)$ & 0.311 \\
\hline Ventilator-free days on day 60 & $0(0-20.4)$ & $8.3(0-40.5)$ & 0.04 \\
\hline Hospital mortality, $n$ (\%) & $29(70.7 \%)$ & $52(46.8 \%)$ & 0.004 \\
\hline
\end{tabular}




\section{Table 2 (continued)}

Data are presented as mean \pm standard deviation, count or median (interquartile range)

ECMO extracorporeal membrane oxygenation, $\mathrm{FiO}_{2}$ fraction of inspired oxygen, $I C U$ intensive care unit, $M P$ mechanical power, $P a C O_{2}$ partial pressure of carbon dioxide in arterial blood, $\mathrm{PaO}_{2}$ partial pressure of oxygen in arterial blood, $P B W$ predicted body weight, $P E E P$ positive end-expiratory pressure, SOFA Sequential Organ Failure Assessment

and $49.3 \%$ among patients with MP of less than $20 \mathrm{~J} / \mathrm{min}$ (Fig. 2a, b).

\section{Factors associated with hospital mortality}

After adjusting for significant confounding variables, Cox proportional hazard regression models revealed a number of factors that were significantly associated with 90-day hospital mortality: immunocompromised status, ARDS duration before ECMO, mean SOFA score from days 1-3 on ECMO, mean MP alone, mean MP referenced to PBW, and mean MP referenced to compliance from days 1-3 on ECMO. The risk of death was higher among patients with higher MP referenced to compliance during ECMO compared to those with higher MP alone or higher MP referenced to PBW (HR 2.289, 1.060, and 1.004, respectively, all $p<0.05$ ) (Table 4 ). The overall 90-day survival rate was significantly higher among severe ARDS patients with mean $\mathrm{MP} \leqq 14.4 \mathrm{~J} / \mathrm{min}$ from day 1 to 3 on ECMO than among those with mean $\mathrm{MP}>14.4 \mathrm{~J} / \mathrm{min}$ (53.2\% vs. $29.3 \%, p=0.004$, log-rank test) (Fig. 3a), and the overall 90-day survival rate was significantly higher among severe ARDS patients with mean MP referenced to compliance $\leqq 0.53 \mathrm{~J} / \mathrm{min} / \mathrm{ml} / \mathrm{cm} \mathrm{H}_{2} \mathrm{O}$ from day 1 to 3 on ECMO than among those with mean $\mathrm{MP}$ referenced to compliance $>0.53 \mathrm{~J} / \mathrm{min} / \mathrm{ml} / \mathrm{cm} \mathrm{H}_{2} \mathrm{O}$ (70.3\% vs. 36.4\%, $p<0.001$, log-rank test) (Fig. 3b). Mean $\mathrm{MP}>14.4 \mathrm{~J} / \mathrm{min}$ during the first 3 days of ECMO was independently associated with higher hospital mortality (Adjusted HR 2.340 [95\% CI 1.358-4.031]; $p=0.002$ ) (Additional file 3: Table S3), and mean MP referenced to compliance $>0.53 \mathrm{~J} / \mathrm{min} / \mathrm{ml} / \mathrm{cm} \mathrm{H}_{2} \mathrm{O}$ during the first 3 days of ECMO was independently associated with higher hospital mortality (Adjusted HR 2.238 [95\% CI 1.224-4.094]; $p=0.009$ ) (Additional file 4: Table S4).

\section{Discussion}

The primary insight in this research was that MP alone, $\mathrm{MP}$ referenced to $\mathrm{PBW}$, and MP referenced to compliance during the first 3 days of ECMO were all independently associated with hospital mortality. Among the ventilator settings variables, mechanical power referenced to compliance during the first 3 days of ECMO had the greatest predictive value for mortality.

ECMO facilitates the use of ultra-protective ventilation, which allows reductions in the contributors of energy load (i.e., MP) to mitigate further lung injury $[1,2]$. Previous studies have reported that during the first 3 days of
ECMO, higher PEEP [21] and lower driving pressure [16, $22]$ were independently associated with lower mortality. However, there was no clearly defined threshold indicating safe ventilator settings and MP values for patients with severe ARDS undergoing ECMO [2]. In the current study, we found that higher MP values during ECMO (but not before ECMO) were associated with increased mortality. In a Cox regression model, mean MP during the first 3 days of ECMO was independently associated with hospital mortality. Overall, our findings revealed that MP during ECMO could be considered a predictor of survival and should be taken into account in optimizing ventilation.

The energy load (MP) delivered to the lungs is not necessarily evenly distributed. The effects of MP on the respiratory system depend not only on the energy load itself but also on the pathophysiology of the lungs (e.g., functional lung size, proportion of inhomogeneity, and the recruitability) [4-6]. Therefore, MP should be adjusted for functional lung size to reflect the actual amount of energy expected to be delivered to the lungs. Specific power (SP), defined as power per ventilated lung unit or the power referenced to the dimension of the ventilated lung, should be considered for predicting VILI more precisely [9-11]. The concept of SP is important due to the fact that the "baby lung" of ARDS has smaller capacity functioning lung tissue for gas exchange, and the SP of the baby lung of ARDS far exceeds the lungs of a healthy adult when the same raw power was delivered [9, 23]. Concentrating the entire ventilation workload on a functioning baby lung that shrinks as it sustains injury increases its power exposure and the risk of entering the "VILI vortex". Earlier intervention to minimize ventilatory demand and its associated MP to avoid progressing down the "VILI vortex" is necessary [11].

Respiratory system compliance is correlated directly with the amount of aerated lung available for tidal ventilation (functional lung size) in patients with ARDS, reflecting the dimension of baby lung [9, 11, 24]. Zhang et al. reported that MP referenced to compliance had highest discrimination in predicting mortality among all ventilator settings variables including MP alone in patients with ARDS [7]. Coppola et al. reported no causal relationship between MP alone and mortality, whereas both MP and transpulmonary MP referenced to respiratory system compliance or to the amount of well-aerated tissue were independently associated with ICU mortality 
Table 3 Ventilator settings, clinical variables, and outcomes as a function of mechanical power/compliance during ECMO

\begin{tabular}{|c|c|c|c|}
\hline \multirow[t]{2}{*}{ Variables } & \multicolumn{2}{|c|}{ MP/Compliance during the first 3 days of ECMO } & \multirow[t]{2}{*}{$p$} \\
\hline & $\begin{array}{l}\operatorname{High}(n=88) \\
\left(>0.53 \mathrm{~J} / \mathrm{min} / \mathrm{ml} / \mathrm{cm} \mathrm{H}_{2} \mathrm{O}\right)\end{array}$ & $\begin{array}{l}\text { Low }(n=64) \\
\left(\leq 0.53 \mathrm{~J} / \mathrm{min} / \mathrm{ml} / \mathrm{cm} \mathrm{H}_{2} \mathrm{O}\right)\end{array}$ & \\
\hline \multicolumn{4}{|l|}{ Ventilator settings before ECMO } \\
\hline $\mathrm{MP}(\mathrm{J} / \mathrm{min})$ & $23.8 \pm 8.7$ & $23.7 \pm 10.1$ & 0.990 \\
\hline MP/PBW $\left(\times 10^{-3} \mathrm{~J} / \mathrm{min} / \mathrm{kg}\right)$ & $422 \pm 158$ & $407 \pm 186$ & 0.583 \\
\hline MP/Compliance $\left(\mathrm{J} / \mathrm{min} / \mathrm{ml} / \mathrm{cm} \mathrm{H}_{2} \mathrm{O}\right)$ & $1.48 \pm 0.85$ & $0.98 \pm 0.49$ & $<0.001$ \\
\hline Tidal volume (ml/kg PBW) & $7.5 \pm 2.4$ & $8.0 \pm 2.3$ & 0.193 \\
\hline PEEP $\left(\mathrm{cm} \mathrm{H}_{2} \mathrm{O}\right)$ & $12.2 \pm 2.8$ & $11.9 \pm 2.7$ & 0.539 \\
\hline Peak inspiratory pressure $\left(\mathrm{cm} \mathrm{H}_{2} \mathrm{O}\right)$ & $35.7 \pm 7.0$ & $31.7 \pm 4.8$ & $<0.001$ \\
\hline Mean airway pressure $\left(\mathrm{cm} \mathrm{H}_{2} \mathrm{O}\right)$ & $19.8 \pm 4.3$ & $17.3 \pm 3.9$ & 0.001 \\
\hline Dynamic compliance $\left(\mathrm{ml} / \mathrm{cm} \mathrm{H}_{2} \mathrm{O}\right)$ & $19.3 \pm 9.4$ & $27.1 \pm 12.2$ & $<0.001$ \\
\hline Total respiratory rate (breaths/min) & $25.2 \pm 7.2$ & $22.8 \pm 6.5$ & 0.042 \\
\hline Spontaneous respiratory rate (breaths/min) & $2(0-8)$ & $0(0-6)$ & 0.197 \\
\hline Minute ventilation (L/min) & $10.4 \pm 3.6$ & $10.8 \pm 3.9$ & 0.536 \\
\hline \multicolumn{4}{|l|}{ Arterial blood gas before ECMO } \\
\hline $\mathrm{pH}$ & $7.27 \pm 0.15$ & $7.28 \pm 0.13$ & 0.627 \\
\hline $\mathrm{PaCO}_{2}(\mathrm{~mm} \mathrm{Hg})$ & $55.5 \pm 21.6$ & $48.1 \pm 14.3$ & 0.014 \\
\hline $\mathrm{PaO}_{2}(\mathrm{~mm} \mathrm{Hg})$ & $74.4 \pm 42.8$ & $71.7 \pm 35.0$ & 0.687 \\
\hline Saturation (\%) & $83.7 \pm 15.4$ & $85.5 \pm 15.5$ & 0.493 \\
\hline $\mathrm{PaO}_{2} / \mathrm{FiO}_{2}(\mathrm{~mm} \mathrm{Hg})$ & $60.7(51.6-83)$ & $67.4(52.9-93.5)$ & 0.851 \\
\hline SOFA score before ECMO & $11.4 \pm 3.2$ & $9.9 \pm 2.9$ & 0.007 \\
\hline \multicolumn{4}{|l|}{ Ventilator settings from day 1 to day 3 on ECMO } \\
\hline $\mathrm{MP}(\mathrm{J} / \mathrm{min})$ & $14.2 \pm 6.8$ & $8.7 \pm 2.9$ & $<0.001$ \\
\hline $\mathrm{MP} / \mathrm{PBW}\left(\times 10^{-3} \mathrm{~J} / \mathrm{min} / \mathrm{kg}\right)$ & $249 \pm 124$ & $149 \pm 51$ & $<0.001$ \\
\hline MP/Compliance $\left(\mathrm{J} / \mathrm{min} / \mathrm{ml} / \mathrm{cm} \mathrm{H}_{2} \mathrm{O}\right)$ & $0.99 \pm 0.45$ & $0.38 \pm 0.10$ & $<0.001$ \\
\hline Tidal volume (ml/kg PBW) & $6.0 \pm 2.3$ & $6.0 \pm 2.1$ & 0.834 \\
\hline PEEP $\left(\mathrm{cm} \mathrm{H}_{2} \mathrm{O}\right)$ & $11.5 \pm 3.2$ & $12.9 \pm 3.2$ & 0.016 \\
\hline Peak inspiratory pressure $\left(\mathrm{cm} \mathrm{H}_{2} \mathrm{O}\right)$ & $34.8 \pm 5.1$ & $27.8 \pm 3.1$ & $<0.001$ \\
\hline Mean airway pressure $\left(\mathrm{cm} \mathrm{H}_{2} \mathrm{O}\right)$ & $18.4 \pm 4.2$ & $16.9 \pm 3.8$ & 0.027 \\
\hline Dynamic compliance $\left(\mathrm{ml} / \mathrm{cm} \mathrm{H}_{2} \mathrm{O}\right)$ & $15.6 \pm 6.7$ & $24.1 \pm 7.3$ & $<0.001$ \\
\hline Total respiratory rate (breaths/min) & $17.9 \pm 4.1$ & $13.1 \pm 3.0$ & $<0.001$ \\
\hline Spontaneous respiratory rate (breaths/min) & $2(0-5)$ & $0(0-3)$ & 0.002 \\
\hline Minute ventilation (L/min) & $6.3 \pm 3.0$ & $4.5 \pm 1.7$ & $<0.001$ \\
\hline \multicolumn{4}{|l|}{ Arterial blood gas from day 1 to day 3 on ECMO } \\
\hline $\mathrm{pH}$ & $7.43 \pm 0.08$ & $7.45 \pm 0.08$ & 0.192 \\
\hline $\mathrm{PaCO}_{2}(\mathrm{~mm} \mathrm{Hg})$ & $39.2 \pm 5.6$ & $37.0 \pm 4.7$ & 0.016 \\
\hline $\mathrm{PaO}_{2}(\mathrm{~mm} \mathrm{Hg})$ & $96.9 \pm 54.0$ & $99.6 \pm 40.9$ & 0.737 \\
\hline Saturation (\%) & $94.6 \pm 3.4$ & $96.3 \pm 2.0$ & $<0.001$ \\
\hline $\mathrm{PaO}_{2} / \mathrm{FiO}_{2}(\mathrm{~mm} \mathrm{Hg})$ & $161.3(125.5-208.8)$ & $203(152.3-250)$ & 0.215 \\
\hline SOFA score from day 1 to day 3 on ECMO & $10.1 \pm 2.3$ & $8.9 \pm 2.2$ & 0.002 \\
\hline ECMO complications, $n(\%)$ & $26(29.5 \%)$ & $17(26.6 \%)$ & 0.688 \\
\hline Duration of ECMO (days) & $10.6(5.1-17.9)$ & $7.9(5.3-12.9)$ & 0.054 \\
\hline Duration of mechanical ventilator (days) & $24(12-42.8)$ & $20(12-34.5)$ & 0.622 \\
\hline Length of ICU stay (days) & $26(14-47)$ & $23(15.5-41)$ & 0.806 \\
\hline Length of hospital stay (days) & $44(18.3-68.3)$ & $38(24.5-65.5)$ & 0.577 \\
\hline ECMO-free days on day 28 & $0(0-15.7)$ & $17.3(0-22.1)$ & $<0.001$ \\
\hline Ventilator-free days on day 28 & $0(0-0)$ & $0(0-15.1)$ & $<0.001$ \\
\hline Ventilator-free days on day 60 & $0(0-25.5)$ & $30.7(0-47.1)$ & $<0.001$ \\
\hline Hospital mortality, $n$ (\%) & $56(63.6 \%)$ & $19(29.7 \%)$ & $<0.001$ \\
\hline
\end{tabular}


Table 3 (continued)

Data are presented as mean \pm standard deviation, count or median (interquartile range)

ECMO extracorporeal membrane oxygenation, $\mathrm{FiO}_{2}$ fraction of inspired oxygen, $I \mathrm{CU}$ intensive care unit, $\mathrm{MP}_{\mathrm{P}}$ mechanical power, $\mathrm{PaCO} \mathrm{C}_{2}$ partial pressure of carbon dioxide in arterial blood, $\mathrm{PaO}_{2}$ partial pressure of oxygen in arterial blood, $P B W$ predicted body weight, $P E E P$ positive end-expiratory pressure, SOFA Sequential Organ Failure Assessment

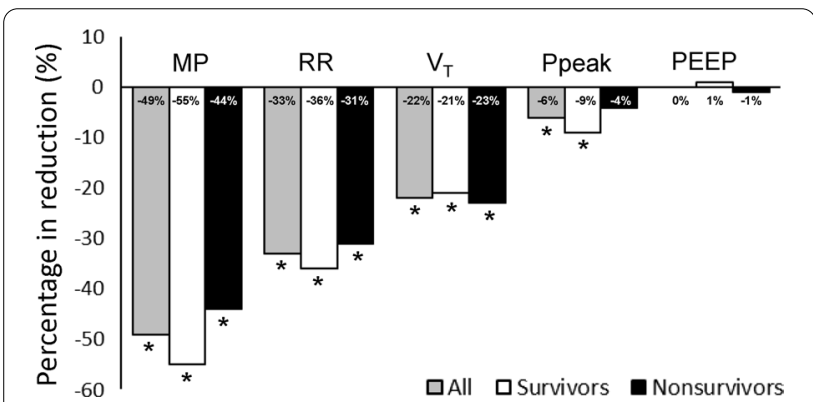

Fig. 1 Difference in mean values of MP and its determinants before and during the first 3 days of ECMO. ${ }^{*} p<0.001$ compared between the mean values before ECMO and during the first 3 days of ECMO. ECMO, extracorporeal membrane oxygenation; MP, mechanical power; PEEP, positive end-expiratory pressure; Ppeak, peak inspiratory pressure; $R R$, respiratory rate; $V_{T}$, tidal volume

of ARDS patients [8]. However, the above studies were predicated on baseline MP values referenced to compliance, they did not account for serial changes in MP referenced to compliance during the ICU stay and did not seek to determine whether the link between MP referenced to compliance and mortality was independent from other ventilator settings.

Patients with severe ARDS requiring ECMO tended to have more noninflated tissue (i.e., lower functional lung size), greater inhomogeneity, and greater lung recruitability [25]. There have been relatively few studies examining the effects of MP referenced to functional lung size on mortality in severe ARDS patients receiving ECMO. In the current study, we found that higher MP/Compliance values during ECMO were significantly associated with increased mortality. Cox regression models revealed that the risk of death estimates obtained using MP referenced to compliance were higher than those of MP alone or MP referenced to PBW, despite the fact that all three factors were independently associated with mortality (HR $2.289,1.060$, and 1.004 , respectively, all $p<0.05)$. It indicated that functional lung size in ARDS patients is not always proportional to body weight [26], and is generally determined by the severity of the disease and is therefore better quantified by compliance $[23,24]$. Our findings demonstrated that MP referenced to compliance is a superior representation of the actual amount of energy transmitted to the lungs and provided the most predictive value for hospital mortality among the ventilatory variables.

The most common cause of death among ARDS patients is multiorgan failure [27]. One international multicenter prospective study reported that extrapulmonary organ failure during ECMO had a significantly negative impact on 6-month mortality for patients with ARDS [19]. Our findings revealed that there was no significant difference between survivors and nonsurvivors in terms of MP and SOFA score before ECMO; however, MP and SOFA score were shown to decrease during the first

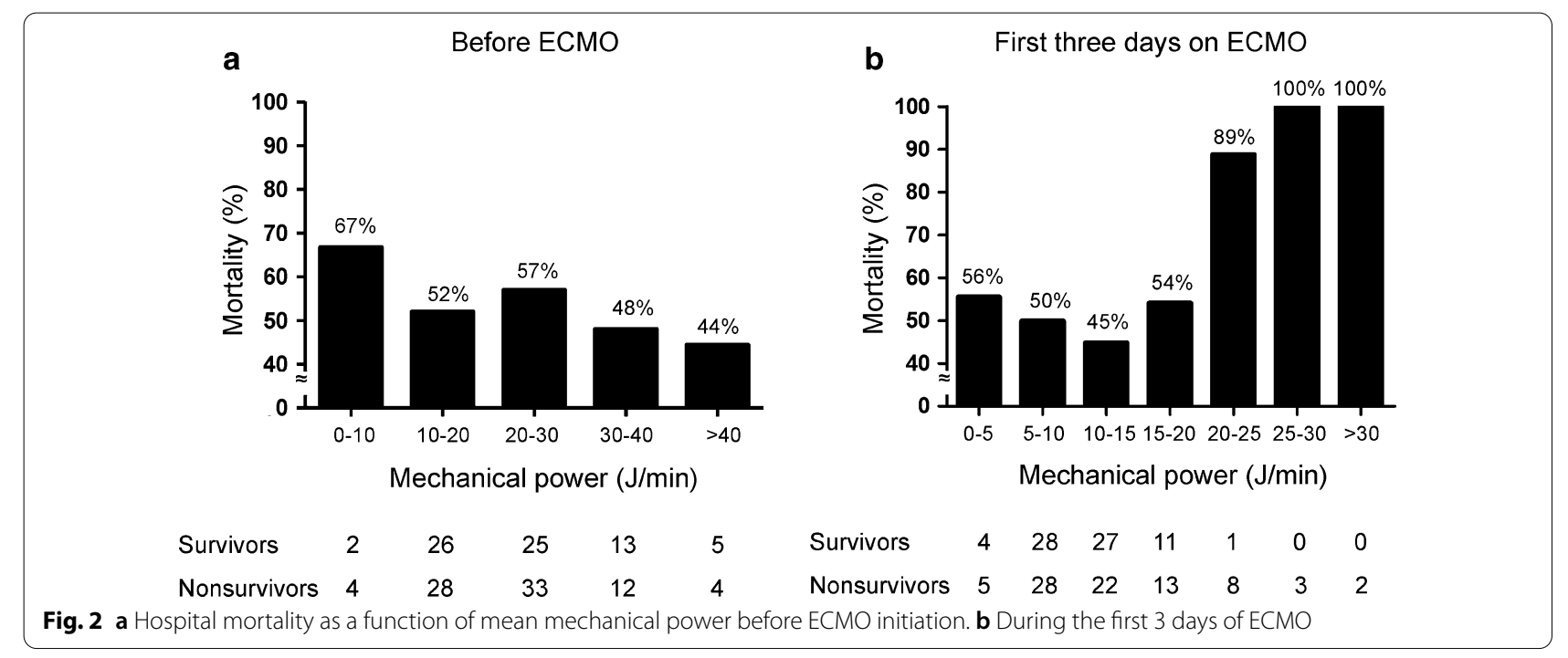


Table 4 Cox proportional hazard regression analysis of factors associated with 90-day hospital mortality

\begin{tabular}{|c|c|c|c|c|c|c|c|c|}
\hline \multirow[t]{2}{*}{ Variables } & \multicolumn{2}{|l|}{ Univariate analysis } & \multicolumn{2}{|c|}{$\begin{array}{l}\text { Multivariate analysis } \\
\text { model } 1\end{array}$} & \multicolumn{2}{|c|}{$\begin{array}{l}\text { Multivariate analysis } \\
\text { model } 2\end{array}$} & \multicolumn{2}{|c|}{$\begin{array}{l}\text { Multivariate analysis } \\
\text { model } 3\end{array}$} \\
\hline & $\mathrm{HR}(95 \% \mathrm{Cl})$ & $p$ & $\mathrm{HR}(95 \% \mathrm{CI})$ & $p$ & $\mathrm{HR}(95 \% \mathrm{Cl})$ & $p$ & $\mathrm{HR}(95 \% \mathrm{CI})$ & $p$ \\
\hline Age (with each year increase) & $1.018(1.004-1.033)$ & 0.012 & & & & & & \\
\hline Pulmonary cause & $1.989(1.211-3.216)$ & 0.007 & & & & & & \\
\hline Extrapulmonary cause & $0.785(0.475-1.296)$ & 0.344 & & & & & & \\
\hline Diabetes mellitus & $0.622(0.358-1.079)$ & 0.091 & & & & & & \\
\hline Chronic liver disease & $2.085(1.184-3.670)$ & 0.011 & & & & & & \\
\hline Immunocompromised status & $2.242(1.411-3.563)$ & 0.001 & $2.564(1.488-4.419)$ & 0.001 & $2.674(1.556-4.596)$ & $<0.001$ & $2.554(1.471-4.433)$ & 0.001 \\
\hline $\begin{array}{l}\text { ARDS duration before ECMO } \\
\text { (with each hour increase) }\end{array}$ & $1.002(1.001-1.004)$ & $<0.001$ & $1.002(1.001-1.004)$ & 0.003 & $1.002(1.001-1.004)$ & 0.003 & $1.001(1.000-1.003)$ & 0.074 \\
\hline $\begin{array}{l}\text { SOFA score from day } 1 \text { to } 3 \\
\text { on ECMO (with each point } \\
\text { increase) }\end{array}$ & $1.318(1.178-1.476)$ & $<0.001$ & $1.202(1.067-1.355)$ & 0.003 & $1.207(1.074-1.356)$ & 0.002 & $1.222(1.084-1.377)$ & 0.001 \\
\hline $\begin{array}{l}\text { Tidal volume/PBW from day } 1 \text { to } \\
3 \text { on ECMO }\end{array}$ & $1.001(0.896-1.118)$ & 0.992 & & & & & & \\
\hline PEEP from day 1 to 3 on ECMO & $0.945(0.880-1.015)$ & 0.120 & & & & & & \\
\hline $\begin{array}{l}\text { Peak inspiratory pressure from } \\
\text { day } 1 \text { to } 3 \text { on ECMO }\end{array}$ & $1.058(1.019-1.100)$ & 0.004 & & & & & & \\
\hline $\begin{array}{l}\text { Dynamic compliance from day } 1 \\
\text { to } 3 \text { on ECMO }\end{array}$ & $0.953(0.924-0.984)$ & 0.003 & & & & & & \\
\hline $\begin{array}{l}\text { Total respiratory rate from day } 1 \\
\text { to } 3 \text { on ECMO }\end{array}$ & $1.055(1.003-1.109)$ & 0.039 & & & & & & \\
\hline MP from day 1 to 3 on ECMO & $1.054(1.017-1.093)$ & 0.004 & $1.060(1.018-1.104)$ & 0.005 & & & & \\
\hline $\begin{array}{l}\text { MP/PBW from day } 1 \text { to } 3 \text { on } \\
\text { ECMO }\left(\times 10^{-3} \mathrm{~J} / \mathrm{min} / \mathrm{kg}\right)\end{array}$ & $1.003(1.001-1.005)$ & 0.002 & & & $1.004(1.002-1.007)$ & $<0.001$ & & \\
\hline $\begin{array}{l}\text { MP/Compliance from day } 1 \text { to } 3 \\
\text { on ECMO }\left(\mathrm{J} / \mathrm{min} / \mathrm{ml} / \mathrm{cm} \mathrm{H}_{2} \mathrm{O}\right)\end{array}$ & $3.142(1.966-5.020)$ & $<0.001$ & & & & & $2.289(1.214-4.314)$ & 0.010 \\
\hline
\end{tabular}

ARDS acute respiratory distress syndrome, $\mathrm{Cl}$ confidence interval, $E C M O$ extracorporeal membrane oxygenation, $H R$ hazard ratio, $M P$ mechanical power, $P B W$ predicted body weight, PEEP positive end-expiratory pressure, SOFA Sequential Organ Failure Assessment

Multivariate analysis models included age, pulmonary or extrapulmonary cause of ARDS, diabetes mellitus, chronic liver disease, immunocompromised status, ARDS duration before ECMO, mean SOFA score from day 1 to 3 on ECMO, and mean values of ventilatory parameters from day 1 to 3 on ECMO (tidal volume/PBW, PEEP, peak inspiratory pressure, dynamic compliance, total respiratory rate, MP, MP/PBW, and MP/Compliance)

Model 1: add mean MP from day 1 to 3 on ECMO

Model 2: add mean MP/PBW from day 1 to 3 on $\mathrm{ECMO}\left(\times 10^{-3} \mathrm{~J} / \mathrm{min} / \mathrm{kg}\right)$

Model 3: add mean MP/Compliance from day 1 to 3 on ECMO $\left(\mathrm{J} / \mathrm{min} / \mathrm{ml} / \mathrm{cm} \mathrm{H}_{2} \mathrm{O}\right)$

3 days of ECMO. SOFA score during the first 3 days of ECMO remained independently associated with hospital mortality. These findings indicated that ECMO could facilitate a further reduction in ventilator load (i.e., MP) in order to alleviate VILI by reducing the proinflammatory biotrauma response, thereby preventing multi-organ failure and improving survival $[2,28,29]$. Besides, an immunocompromised status was associated with lower survival, as reported in previous studies $[19,30]$. The timing of ECMO initiation for severe ARDS has yet to be defined [1]; however, recent studies have also reported a link between ARDS duration before ECMO and mortality $[19,29]$.

This study was hindered by a number of limitations. First, this retrospective study was conducted in one tertiary care referral center with a high annual volume of patients requiring ECMO, thereby limiting generalizability. Second, ventilatory variables were recorded only once a day during the stay in the ICU and therefore do not necessarily represent dynamic changes in ventilator status, including fluctuations in MP during 24-h intervals. Third, we assessed functional lung size by means of PBW and compliance due to the retrospective study, but computed tomography scan of the lungs may be more accurate way to estimate amount of aerated remaining functional lung, lung inhomogeneity or the recruitability $[7,25]$. However, computed tomography scan requires intra-hospital patient transfer from ICU to radiology department and the use of ECMO preclude widespread clinical use. Finally, our objective in this observational study was to identify the factors associated with mortality without considering issues pertaining to causality. 


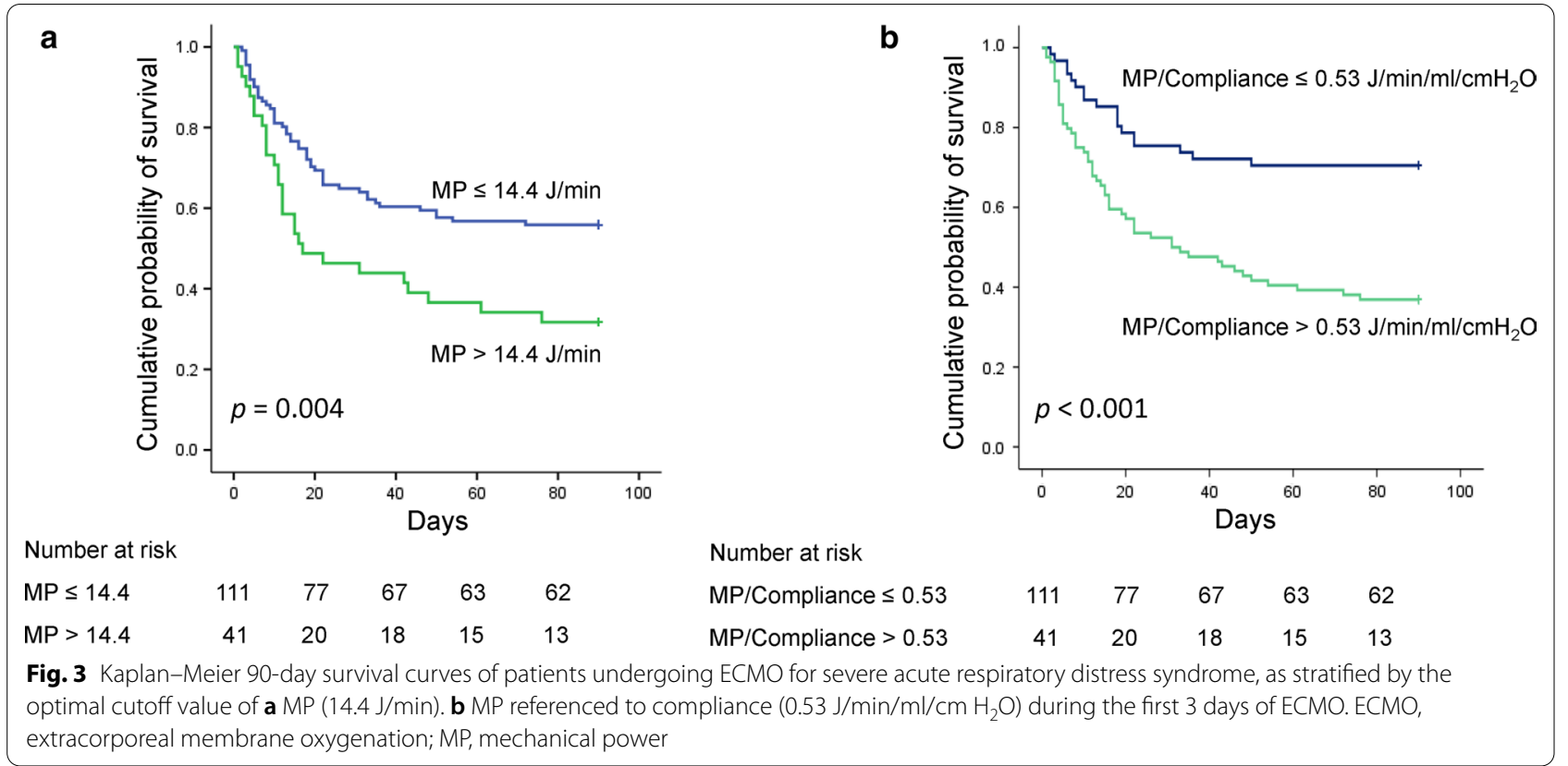

\section{Conclusions}

Our findings revealed that MP referenced to compliance provided the most predictive value for hospital mortality among the ventilator settings variables. Defining safety limits to minimize VILI and decrease mortality in patients with severe ARDS undergoing ECMO may require larger randomized controlled trials to determine whether MP referenced to functional lung size, lung inhomogeneity, or recruitability is causally related to mortality.

\section{Supplementary Information}

The online version contains supplementary material available at https://doi. org/10.1186/s13054-020-03428-x.

Additional file 1: Table S1. Ventilator settings before and during the first 3 days of ECMO between the earlier years and later years.

Additional file 2: Table S2. Ventilator settings parameters during the first 3 days of ECMO

Additional file 3: Table S3. Cox proportional hazard regression analysis of factors associated with 90-day hospital mortality.

Additional file 4: Table S4. Cox proportional hazard regression analysis of factors associated with 90-day hospital mortality.

\section{Abbreviations}

ARDS: Acute respiratory distress syndrome; $\mathrm{Cl}$ : Confidence interval; ECMO: Extracorporeal membrane oxygenation; HR: Hazard ratio; ICU: Intensive care unit; MP: Mechanical power; PBW: Predicted body weight; PEEP: Positive endexpiratory pressure; SOFA: Sequential organ failure assessment; SP: Specific power; $V_{T}$ : Tidal volume; Ppeak: Peak inspiratory pressure; $\triangle P$ : Driving pressure; RR: Respiratory rate; VILI: Ventilator-induced lung injury.

\section{Acknowledgements}

The authors would like to express their appreciation for the patients and staff in the ICU at Chang Gung Memorial Hospital. We thank Mr. Yu-Jr Lin in Research Services Center For Health Information, Chang Gung University for validating and confirming all the statistics in this study.

\section{Authors' contributions}

LCC and KCK assumed responsibility for the accuracy of the data analysis and drafting of the manuscript. LCC, SWL, LPC, HHL, FCT, CHC, and CYH performed the study design and data acquisition. $\mathrm{LCC}$ and $\mathrm{PHL}$ were responsible for statistical analysis of data. LCC, CSL, SWL, HCH, CCH, HPW, and KCK performed interpretation of the results. All authors read and approved the final manuscript.

\section{Funding}

This study was supported by grant CORPG3G0151 from Chang Gung Memorial Hospital.

\section{Availability of data and materials}

The datasets used or analyzed in the study are available from the corresponding author on reasonable request.

\section{Ethics approval and consent to participate}

The local Institutional Review Board for Human Research approved this study (CGMH IRB No. 201600632B0), and the need for informed consent was waived.

\section{Consent for publication}

Not applicable.

\section{Competing interests}

On behalf of all authors, the corresponding author states that there are no conflicts of interest.

\section{Author details}

${ }^{1}$ Department of Thoracic Medicine, Chang Gung Memorial Hospital, Chang Gung University College of Medicine, Linkou, No. 5, Fu-Shing St., GuiShan, Taoyuan, Taiwan. ${ }^{2}$ Graduate Institute of Clinical Medical Sciences, College of Medicine, Chang Gung University, Taoyuan, Taiwan. ${ }^{3}$ Department 
of Thoracic Medicine, New Taipei Municipal TuCheng Hospital and Chang Gung University, Taoyuan, Taiwan. ${ }^{4}$ Department of Respiratory Therapy, Chang Gung University College of Medicine, Taoyuan, Taiwan. ${ }^{5}$ Institute of Emergency and Critical Care Medicine, School of Medicine, National Yang-Ming University, Taipei, Taiwan. ${ }^{6}$ Clinical Informatics and Medical Statistics Research Center, College of Medicine, Chang Gung University, Taoyuan, Taiwan. ${ }^{7}$ Division of Endocrinology and Metabolism, Department of Internal Medicine, Chang Gung Memorial Hospital, Taoyuan, Taiwan. ${ }^{8}$ Division of Cardiovascular Surgery, Chang Gung Memorial Hospital, Taoyuan, Taiwan. ${ }^{9}$ Department of Respiratory Therapy, Chang Gung Memorial Hospital, Chang Gung University College of Medicine, Taoyuan, Taiwan. ${ }^{10}$ Division of Pulmonary, Critical Care and Sleep Medicine, Chang Gung Memorial Hospital, Keelung, Taiwan.

${ }^{11}$ Department of Intensive Care, Xiamen Chang Gung Hospital, Xiamen, China.

Received: 9 October 2020 Accepted: 7 December 2020

Published online: 06 January 2021

\section{References}

1. Brodie D, Bacchetta M. Extracorporeal membrane oxygenation for ARDS in adults. N Engl J Med. 2011;365:1905-14.

2. Abrams D, Schmidt M, Pham T, Beitler JR, Fan E, Goligher EC, et al. Mechanical ventilation for acute respiratory distress syndrome during extracorporeal life support. Research and practice. Am J Respir Crit Care Med. 2020;201:514-25.

3. Cressoni M, Gotti M, Chiurazzi C, Massari D, Algieri l, Amini M, et al. Mechanical power and development of ventilator-induced lung injury. Anesthesiology. 2016;124:1100-8.

4. Gattinoni L, Tonetti T, Cressoni M, Cadringher P, Herrmann P, Moerer O, et al. Ventilator-related causes of lung injury: the mechanical power. Intensive Care Med. 2016;42:1567-75.

5. Gattinoni L, Tonetti T, Quintel M. Intensive care medicine in 2050: ventilator-induced lung injury. Intensive Care Med. 2018;44:76-8.

6. Gattinoni L, Marini JJ, Collino F, Maiolo G, Rapetti F, Tonetti T, et al. The future of mechanical ventilation: lessons from the present and the past. Crit Care. 2017;21:183

7. Zhang Z, Zheng B, Liu N, Ge H, Hong Y. Mechanical power normalized to predicted body weight as a predictor of mortality in patients with acute respiratory distress syndrome. Intensive Care Med. 2019;45:856-64.

8. Coppola S, Caccioppola A, Froio S, Formenti P, De Giorgis V, Galanti V, et al. Effect of mechanical power on intensive care mortality in ARDS patients. Crit Care. 2020;24:246.

9. Marini JJ, Rocco PRM, Gattinoni L. Static and dynamic contributors to ventilator-induced lung injury in clinical practice. Pressure, energy, and power. Am J Respir Crit Care Med. 2020;201:767-74.

10. Collino F, Rapetti F, Vasques F, Maiolo G, Tonetti T, Romitti F, et al. Positive end-expiratory pressure and mechanical power. Anesthesiology. 2019;130:119-30.

11. Marini JJ, Gattinoni L. Time course of evolving ventilator-induced lung injury: the "shrinking baby lung." Crit Care Med. 2020;48:1203-9.

12. Serpa Neto $A$, Deliberato RO, Johnson AEW, Bos LD, Amorim P, Pereira SM, et al. Mechanical power of ventilation is associated with mortality in critically ill patients: an analysis of patients in two observational cohorts. Intensive Care Med. 2018;44:1914-22.

13. Parhar KKS, Zjadewicz K, Soo A, Sutton A, Zjadewicz M, Doig L, et al. Epidemiology, mechanical power, and 3-year outcomes in acute respiratory distress syndrome patients using standardized screening. An observational cohort study. Ann Am Thorac Soc. 2019;16:1263-72.

14. ARDS Definition Task Force, Ranieri VM, Rubenfeld GD, Thompson BT, Ferguson ND, Caldwell E, et al. Acute respiratory distress syndrome: the Berlin Definition. JAMA. 2012;307:2526-33.
15. Schmidt MFS, Amaral ACKB, Fan E, Rubenfeld GD. Driving pressure and hospital mortality in patients without ARDS: a cohort study. Chest. 2018;153:46-54.

16. Chiu LC, Hu HC, Hung CY, Chang CH, Tsai FC, Yang CT, et al. Dynamic driving pressure associated mortality in acute respiratory distress syndrome with extracorporeal membrane oxygenation. Ann Intensive Care. 2017;7:12.

17. Tojo K, Yoshida T, Yazawa T, Goto T. Driving-pressure-independent protective effects of open lung approach against experimental acute respiratory distress syndrome. Crit Care. 2018;22:228.

18. Silva PL, Ball L, Rocco PRM, Pelosi P. Power to mechanical power to minimize ventilator-induced lung injury? Intensive Care Med Exp. 2019;7(Suppl 1):38.

19. Schmidt M, Pham T, Arcadipane A, Agerstrand C, Ohshimo S, Pellegrino $\checkmark$, et al. Mechanical ventilation management during extracorporeal membrane oxygenation for acute respiratory distress syndrome. An international multicenter prospective cohort. Am J Respir Crit Care Med. 2019;200:1002-12.

20. Urner M, Jüni $P$, Hansen B, Wettstein MS, Ferguson ND, Fan E. Timevarying intensity of mechanical ventilation and mortality in patients with acute respiratory failure: a registry-based, prospective cohort study. Lancet Respir Med. 2020;8:905-13.

21. Schmidt M, Stewart C, Bailey M, Nieszkowska A, Kelly J, Murphy L, et al. Mechanical ventilation management during extracorporeal membrane oxygenation for acute respiratory distress syndrome: a retrospective international multicenter study. Crit Care Med. 2015;43:654-64.

22. Serpa Neto A, Schmidt M, Azevedo LC, Bein T, Brochard L, Beutel G, et al. Associations between ventilator settings during extracorporeal membrane oxygenation for refractory hypoxemia and outcome in patients with acute respiratory distress syndrome: a pooled individual patient data analysis: Mechanical ventilation during ECMO. Intensive Care Med. 2016;42:1672-84

23. Gattinoni L, Marini JJ, Pesenti A, Quintel M, Mancebo J, Brochard L. The, "baby lung" became an adult. Intensive Care Med. 2016;42:663-73.

24. Amato MB, Meade MO, Slutsky AS, Brochard L, Costa EL, Schoenfeld DA, et al. Driving pressure and survival in the acute respiratory distress syndrome. N Engl J Med. 2015;372:747-55.

25. Maiolo G, Collino F, Vasques F, Rapetti F, Tonetti T, Romitti F, et al. Reclassifying acute respiratory distress syndrome. Am J Respir Crit Care Med. 2018;197:1586-95.

26. Chiumello D, Carlesso E, Brioni M, Cressoni M. Airway driving pressure and lung stress in ARDS patients. Crit Care. 2016;20:276.

27. Thompson BT, Chambers RC, Liu KD. Acute respiratory distress syndrome. N Engl J Med. 2017;377:562-72.

28. Curley GF, Laffey JG, Zhang H, Slutsky AS. Biotrauma and ventilatorinduced lung injury: clinical implications. Chest. 2016;150:1109-17.

29. Chiu LC, Tsai FC, Hu HC, Chang CH, Hung CY, Lee CS, et al. Survival predictors in acute respiratory distress syndrome with extracorporeal membrane oxygenation. Ann Thorac Surg. 2015;99:243-50.

30. Schmidt M, Schellongowski P, Patroniti N, Taccone FS, Reis Miranda D, Reuter J, et al. Six-month outcome of immunocompromised patients with severe acute respiratory distress syndrome rescued by extracorporeal membrane oxygenation. An international multicenter retrospective study. Am J Respir Crit Care Med. 2018;197:1297-307.

\section{Publisher's Note}

Springer Nature remains neutral with regard to jurisdictional claims in published maps and institutional affiliations. 